\title{
Hiperlipoproteinemia tipo I
}

\author{
Dra. Patricia Bustos $M{ }^{1}$; Dra. Eliana Fernández $S^{2}{ }^{2}$; \\ Dra. M. Fugenia Radrigán K.
}

Type hyperlipoproteinemia

\begin{abstract}
A case of type I hyperlipoproteinemia wich has been followed up for 5 years is reported. Clinical manifestations (eruptive xanthoma) appeared at 3 month of age. Plasma analysis revealed marked hypertriglyceridemia and chylomicronemia. Xanthoma dissapeated and blood trigfycerides concentration decressed following gradual reduction of Eat intake. Maintenance on low lipid diet for extended periods is the main difficulty in the management of this unf requent metabolic disorder.

(Key words: type I hyperlipoproteinemia, eruptive xanthoma, hypertriglyceridemia, chy lomicronemia, low fat diet). .
\end{abstract}

La hiperlipoproteinemia (HIP) tipo I es poco frecuente, se diagnostica generalmente en la niñez y que se caracteriza por la presencia de xantoma eruptivo, dolor abdominal y lipemia retinalis $1,2,3$. Esta enfermedad se debe a una insuficiente remoción de los quilomicrones del plasma por inhabilidad de la enzima lipoproteica para hidrolizar las lipoprote inas ricas en triglicêridos. Esta disminución de la actividad enzimátíca puede ser secundaria a bajos niveles de la enzima (en su forma clásica) ${ }^{4}$, a una deficiencia de la apolipoproténa $\mathrm{C}$-II que es un cofactor que requiere la enzima para su actividad ${ }^{5}$ o a la presencia de un inhibidor de la actividad enzimática en el plasma ${ }^{6}$.

La apariencia del plasma después de dejarlo en reposo a baja temperatura, orienta el diagnóstico. También son de ayuda el aumento de los triglicéridos plasmáticos $\mathrm{y}$ un patrón tipo I (quilomicronemia) en la electroforesis de lípidos. Sin embargo, el ideal para hacer el diagnóstico es que se precise la deficiencia bioquímica involucrada $^{7}$.

El tratamiento consiste en la disminución del aporte de lípidos de la dieta a un nivel tan bajo

1. Departamento de Nutrición, Facultad de Medicina, Unjversidad de Chile.

2. Departamento y Servicio de Pediatría, Hospital Roberto del Río, Universidad de Chile. que haga al paciente asintomático:0,5g de grasa $x$ $\mathrm{kg} x$ día o menos en adultos y $10 \mathrm{~g}$ de grasa $x$ día en niños, debiendo reducirse tanto el aporte de lípidos saturados como poliinsaturados. Se aconseja la administración de ácidos grasos de cadena media ya que estos se absorben directamente sin formar quilomicrones ${ }^{8,9}$.

El diagnóstico diferencial de la HLP tipo I se debe hacer con la HIP tipo $\mathrm{V}$, que también se presenta con hiperquilomicronemia pero, a diferencia de la tipo I, es frecuente en adultos y secundaria a otras aiteraciones metabólicas como diabetes y síndrome nefrótico; con la HLP tipo IV que se detecta en los adultos y tiene hipertrigliceridemia pero sin quilomicronemia y con la HIP tipo II que, como el tipo I se presenta en la nif́ez con xantomas pero, además, con hipercolesterolemia 10-13.

E1 caso descrito a continuación, en consideración a su baja frecuencia, ilustra la buena respuesta de la enfermedad al tratamiento dietético.

\section{Caso clinico}

Lactante de dos y medio meses, referida al Hospital Roberto del Río por lesiones de la piel que aparecieron una semana después del nacimiento, que consistían en múltiples pápulas duras, pequeñas ( 1 a $4 \mathrm{~mm}$ de diámetro) de color blanquecino con leve fondo eritematoso ubicadas 


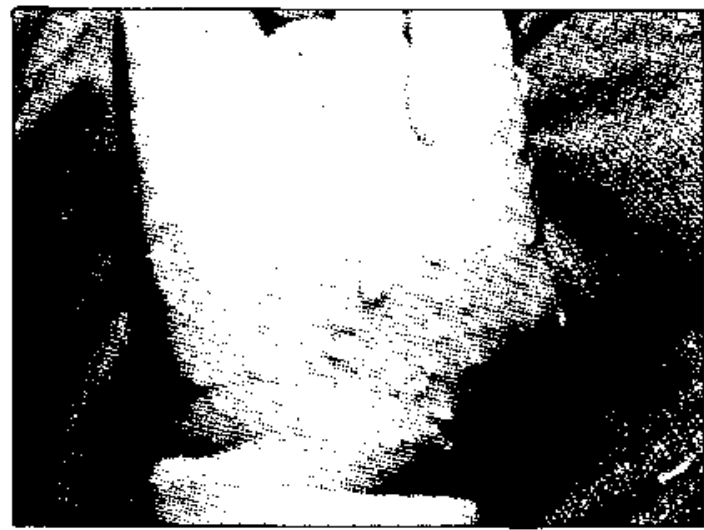

Figura 1. Hiperlipoproteinenia tipo I: pápulas de xantoma cruptivo en mano.

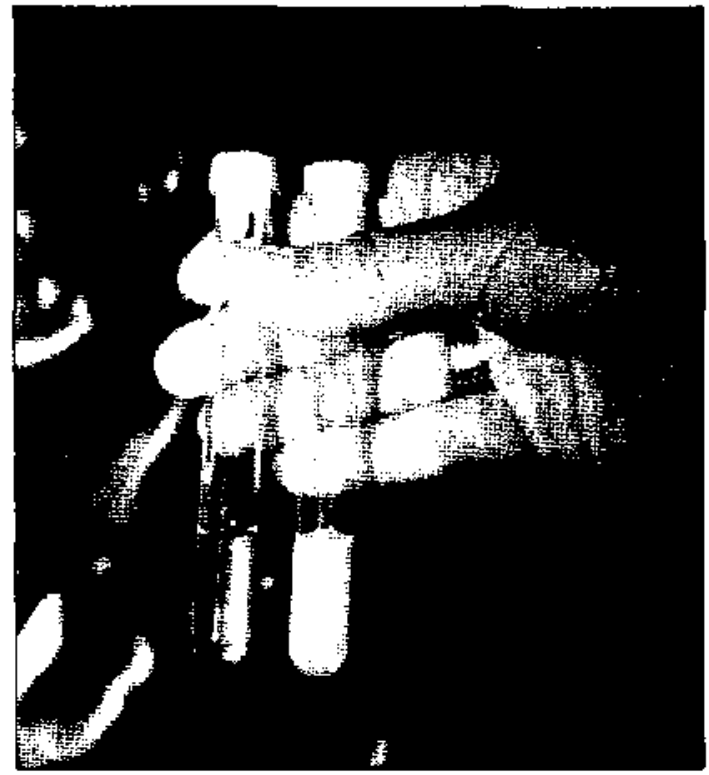

Figura 2. Plasma de coloración blanco lechosa de la hiperlipoproteinemia tipo I, comparado con plasma normal.

en paladar, mejitlas y dorso de manos y pies (figura 1). Borde inferior del higado $2 \mathrm{~cm}$ bajo reborde; relación peso/talla $114 \%$ porcentaje de grasa magra $28 \%$. La biopsia de una de las pápulas, fue compatible con el diagnóstico clínico de xantoma eruptivo, La concentración sanguínea de triglicéridos era $2.640 \mathrm{mg} \mathrm{x} \mathrm{dl} \mathrm{(} \mathrm{n}=50)$ $-120 \mathrm{mg} x \mathrm{dl}$ ) y la de colesterol $537 \mathrm{mg} \mathrm{x} \mathrm{dl}$ ( $n=180-240 \mathrm{mg} \times \mathrm{dl}$ ).

Inicialmente se le restringió el aporte de calorías para disminuir los lipidos sanguineos,

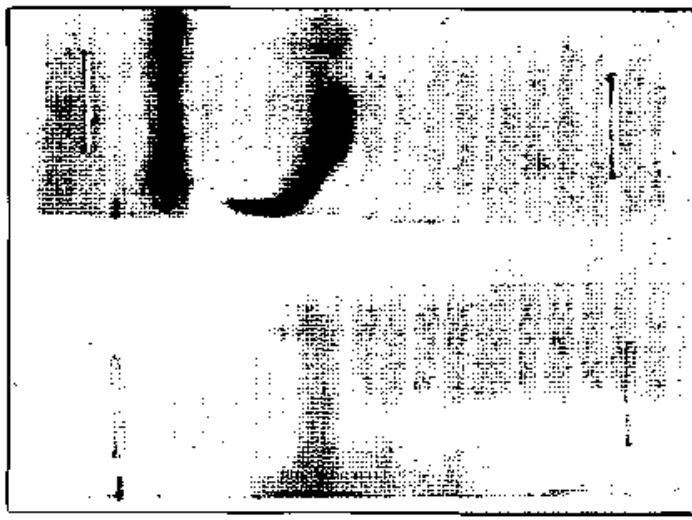

Figura 3. Vjectroforexis de lipoproteínas: placa superior, cipo I en que se observa una banda de alta densidad en el comienzo de la migración (corresponde a suilomicro. nes). Placa inferior, normal.

mediante leche de vaca reconstituída con azúcar $(600 \mathrm{cc})$, almuerzo y postre, alimentación que le proporcionó $95 \mathrm{Cal} \times \mathrm{kg} \times$ dia, un aporte de grasas de $3 \mathrm{~g} \times \mathrm{kg} \times$ dia $y$ un $\mathrm{G} \%$ de 30 . Pocos días después de su ingreso una nueva muestra de plasma llamó la atención por su coloración blanco lechosa (figura 2) y porque dejada en reposo se separó en dos capas, siendo la superior identificada como quilomicrones. La medición de lípidos sanguíneos realizada en el laboratorio de Nutrición de la Universidad Católica dio los resultados siguientes', triglicéridos $640 \mathrm{mg} \mathrm{x} \mathrm{dl}$, colesterol $145 \mathrm{mg} x$ di $y$ electroforesis de lipoproteínas con patrón tipo I (figura 3) precisándose que se trataba de una hiperlipoproteinemia tipo ].

Debido a que la pancreatitis por hipertrigliceridemia eș la complicación más grave de esta afección, se propuso reducir los triglicéridos de la dieta, empleándose para este fin una leche con bajo contenido de grasa (1\%) diluida al $7,5 \%$, con agregado de aceite de maíz al $2 \%$, azúcar al $5 \%$, crema de arroz al $5 \%$ y alimentación sólida con bajo contenido en lípidos. Con esta dieta cambiaba el típo de grasas, restringiendose la grasa láctea de la fórmula anterior (que daba fundamentalmente ácidos grasos saturados), aportándose ahora lípidos poliinsaturados con el agregado de aceite de la fórmuna láctea. El G\% se redujo a casi la mitad del proporcionado al comienzo manteniéndose el aporte de ácido linoleico en $1 \%$ de las calorías. Después de 12 dias con esta dieta de prueba se controlaron los lipidos sanguíneos: paradojalmente los valores 
obtenidos fueron colesterol $202 \mathrm{mg} x$ dl y triglicéridos $1.189 \mathrm{mg} \mathrm{x}$ dl. En vista de estos resultados, se modificó nuevamente la alimentación, disminuyendo el aporte de aceite de la fórmula (al $1 \%$ y conservando la alımentación sólida. De este modo se estableció un aporte de grasa de un tercio del inicial, con $1 \mathrm{~g}$ de grasa por kilo por día y $G \%$ de 10 , dado casi en su totalidad por aceite de máz, que proporciona fundamentalmente ácidos grasos esenciales. Una semana después de indicada esta tercera dieta, el plasma habia adquirido coloración normal y las concentraciones sanguíneas de lípidos eran me. notes: colesterol, $146 \mathrm{mg} \%$ y triglicéridos 131 ing $\%$.

Esta paciente ha sido seguida durante 5 años evidenciándose un crecimiento y desarrollo normales. En relación a las transgresiones de la dieta, han aparecido xantomas especialmente en las manos y las cifras de colesterol y triglicéridos han estado permanentemente elevadas (tabla). Influye en esta evolución que la niña asiste a jardin infantil y además es capaz por sí sola de proporcionarse los alimentos que Je están prohi. bidos.

\section{DISCUSION}

Los hechos que orientaron el diagnóstico en esta paciente fueron el xantoma cruptivo, la hiperlipoprotenemia y la desaparición de ésta después de disminuir los lípidos de la dieta, lo que caracteriza a la HLP tipo I.Llama la atención la precocidad con que aparecieron los síntomas lo que hace pensar que se debió a la ingesta de leche materna, rica en grasas. Probablemente no se detectó dolor abdominal en esta paciente por la dificultad para pesquisarlo en los lactantes menores o por la precocidad con que se restringió la grasa en la dieta. No se buscó lipemia retinalis por ser esta una característica inespecifica'de la enfermedad $y$ por el riesgo de exponer a un lactante a anestesia general para realizar un fondo de ojo.

Por no contar en nuestro país con la téc. nica para la determinación enzimática idealmente necesaria para el diagnóstico, Este se basó en hechos clínicos: una muestra de plasna lechoso con valores de triglicéridos muy aumentados y una electroforesis de lípidos tipo I. Fue necesario introducir sucesivas modificaciones de la alimentación hasta llegar a un aporte de lípidos que mantuviera bajos los triglicéridos, empleando aceite de maíz. A pesar de ser éste una buerra fuente de ácidos grasos esenciales en este paciente, ellos quedaron ligeramente bajo to recomendado debido al nivel de reducción del aporte de grasa al que debió recurrirse. Es aconsejable, además, agregar diariamente vitaminas; liposolubles, debido a que por e1 bajo aporte de lípidos, la absorción de estas vitaminas está disminuida.

\section{RESUMEN}

Se presenta el caso clínico de una paciente portadora de una hiperlipoproteinemia tipo I controlada durante 5 arios. Se describe las características clínicas y de laboratorio de esta enfermedad así como la respuesta al tratamiento dietético que es fundamental en la evolución de esta alteración metabólica tan poco frecuente.

Tabla

Hiperlipoproteinemia tipo I: Evolución de los lípidos sanguíneos

\begin{tabular}{lcccccccc}
\hline EDAD & $\begin{array}{c}2 \\
\text { meses }\end{array}$ & $\begin{array}{c}3 \\
\text { meses }\end{array}$ & $\begin{array}{c}4 \\
\text { meses }\end{array}$ & $\begin{array}{c}5 \\
\text { meses }\end{array}$ & $\begin{array}{c}8 \\
\text { meses }\end{array}$ & $\begin{array}{c}18 \\
\text { meses }\end{array}$ & $\begin{array}{c}4 \text { anos medio } \\
\text { años }\end{array}$ \\
\hline $\begin{array}{l}\text { Colesterol (mg } \\
\text { rango normal=50-220 }\end{array}$ & 537 & 145 & 202 & 146 & 160 & 200 & 313 & 252 \\
$\begin{array}{l}\text { Triglicéridos (mg!) } \\
\text { rango normal }=40-140\end{array}$ & 2640 & 640 & 1189 & 313 & 420 & 480 & 1061 & 1455 \\
$\begin{array}{l}\text { Electroforesis de } \\
\text { tipoproteinas }\end{array}$ & Tipo I
\end{tabular}




\section{AGRADECIMIENTOS:}

Al Dt. Antonio Atteaga Ll. por su valiosa colaboración en la formulación del diagnóstico, así como en el análisis del passna en el Laboratorio de nutrición de ta Universidad Católica.

\section{REFERENCIAS}

1. Fredrickson $D_{1}$, Goldstein $J$. , Brown $M .:$ The Familial Hyperlipopr oteinemias. In the Metabolic Basis of Inherited Disease. Stambury.; Wyngaarden, J., and Fredricksón. D.: $4^{\circ}$ Ed. New York. McGraw Hill Book Co. 1978; 551-564.

2. Rausen A., Adlersberg D.: Idiopatic Hyperlipenia and Hypercholesterolemia in Children. Pediatrics 1961; 28: 276-284.

3. Thannhauser S.: Xantomatoses. In Lipidoses. Grune and Stratton. N. York, $1958 ; 40-47$.

4. Feuer $G$., $D e$ la Iglesio $F$.: Abnormalities of lipid synthesis and metabolism. In Molecular Bioche mistry of Hunan Disease. CRC. Press Inc. Florida, 1985; 116-118.

5. Yamamura $T$.: Familial Type I Hyperlipoproteinemia caused by Apoprotein C-II Deficiency. Atherasclerosis $1979 ; 34: 53-58$

6. Brunzell $J$, Muller $N_{\text {. }}$ : Familial Chylomicronemia due to a circulating inhibitor of lipoprotein lipase activity.. I Lipid Res $1983 ; 24: 12-16$.

7. Levy R., Ernst N. : Diet, Hyperlipemia and Atherosclerosis. In Modern Nutrition on Health and Disease, Goodhart, R. and Shils, M. Lea and Febiger. Pliiladelphia, 1980; 1058-1066.

8. Netsont W: Textbook of Pediatrics, 12a.Ed. W.B. Saunders Co. Philadelphía. 1983; 1200-1203.

9. Rifkind B., Levy R. : Hyperlipidemia: Diagnoses and Therapy, Clinical Cardiology Monographs. New York, Grune and Stratton Inc. 1977; 78-89.

10. Commitee on Nutrition, American Academy of Pediatrics. Chapter 27. In Pediatric Nutrition Handbook. Illinois Academy of Pediatrics. 1985; 238-245.

11. Glueck G., Fallat $R$., Tsang R,: Hypercholesterolemia and hypertriglyceridemia in children. Am J Dis Child; $128: 569.574$.

12. Walker A., Natkins $J$ : Hyperlipidemia. In Jounnal of Nutrition in Pediatrics. Basic Science and Clinical Aplication. Boston. Little, Brown and Company. $1985 ; 572-575$.

13. Mauricci A., Noziglia C., Burrows R, Muzzo S.: A propósito de un caso clínico de hiperlipoproteinemia tipo Il A en un preencolar. Rev Chil Nutr 1985; 13: 189-193.

14. Dauncey M.J., Gandy G., Gairdner D.: Assessment of total body fat in infancy from skinfold thickness measurements. Arch Dis Child 1977: 52: 223-227. 